\title{
Response times to Ehrenstein illusions of varying subjective magnitude: Complementarity of psychophysical measures
}

\author{
DELPHINE PINS, CLAUDE BONNET, and BIRGITTA DRESP \\ Université Louis Pasteur, Strasbourg, France
}

\begin{abstract}
The following experiments investigate the effects of contrast polarity, inducer spacing, and inducer type on three dependent variables measuring the perception of an illusory surface in Ehrenstein figures: subjective magnitude, response time, and frequency of perception. It was found that response time generally decreased when the other two behavioral indicators increased. However, it was also shown that subjective magnitude provided more discriminating measures of relatively strong illusory percepts, whereas frequency of perception and response time provided more discriminating measures of relatively weak illusory percepts. The findings generally confirm earlier work on the effect of inducer spacing and contrast polarity on the perceived strength of brightness illusions, and in particular reveal the complementarity of subjective magnitude, response time, and frequency of perception as critical measures of configurational effects in the perceptual processing of these phenomena.
\end{abstract}

Phenomena of illusory form (for a review, see Spillmann \& Dresp, 1995) have been studied and discussed in the literature on visual perception for some time. Illusory forms are generally induced by configurations of black and/or white fragments arranged in the visual field according to well-defined structural principles. The alignment or collinearity of edges or line ends can be considered as the most critical factor, together with the size and the spatial separation of the individual fragments. The Ehrenstein illusion (Figure 1A) as a particular example is characterized by the perception of a bright surface in the center of a stimulus configuration consisting of a set of (usually four) converging lines. The ends of the lines must fall on the same virtual contour axis so that they can be perceptually linked to form either an illusory diamond, a square, or a circle (see again Figure 1A). The brightness phenomenon perceived in these configurations is called "illusory" because it emerges despite the absence of a physical difference in luminance between the apparent figure and the ground. Various experiments have shown that the illusion depends mainly on local characteristics of the stimulus such as the length, the spacing, or the luminance of the inducing lines, indicating that the relative strength of illusory brightness is mainly a function of the physical parameters defining the stimulus or its context. In particular, it has been shown that perceived brightness diminishes when the spatial separation between inducers increases (Lesher \& Mingolla, 1993; Shipley \& Kellman, 1992; Zucker \& Davis, 1988). However, variations in stim-

Thanks are due to J. E. Hoffman, T. Parks, and an anonymous referee for their insightful comments and suggestions on an earlier version of this paper. Correspondence should be addressed to D. Pins, Unité INSERM 405, Département de Psychiatrie, Hôpitaux Universitaires, BP 426, F-67091 Strasbourg, France (e-mail: pins@alsace.u-strasbg.fr). ulus luminance or contrast polarity produce sometimes paradoxical effects (see, e.g., Dresp, Salvano-Pardieu, \& Bonnet, 1996; Spillmann, Fuld, \& Neumeyer, 1984) indicating that the relationship between the physical stimulus and the induced brightness percept is far from being straightforward. Attentional factors and psychological processes related to stimulus uncertainty may explain some of these observations; however, the question of which would be the appropriate method to bring them to the fore remains a critical issue (see, e.g., the review by Spillmann \& Dresp, 1995).

Here, we examine the possibility of linking different psychophysical measures in the investigation of subjective brightness phenomena and discuss how using different methods jointly in a psychophysical experiment may help to overcome the problem of subjectivity that occurs with rating or scaling tasks, which are commonly used to study brightness perception. Dresp et al. (1996) have reported experiments with configurations giving rise to the perception of Ehrenstein illusions with a surprising variation in phenomenal strength. This was achieved by varying the spacing, the length, and the contrast polarity of the inducing element defining the configurational context. The main results of these experiments have shown that the frequency with which the illusion is perceived varies as a function of size--spacing contraints imposed by the inducing elements. Moreover, these results have suggested that surface enhancement in illusory figures is stronger in configurations with inducers of the same contrast polarity, although stimuli where polarity varies from one inducing line to the other to some extent also seem to produce the phenomenon. Collinear inducing elements the polarity of which is not constant within a given line of induction element failed to produce an illusory surface. Finally, continuous lines produced stronger illusions than 


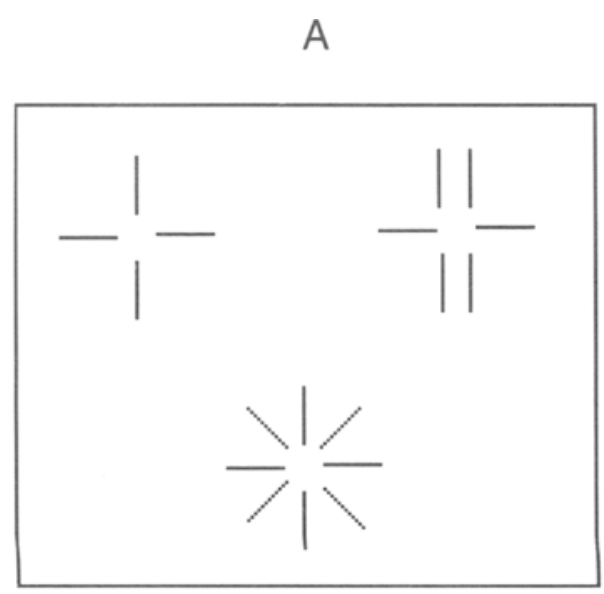

B

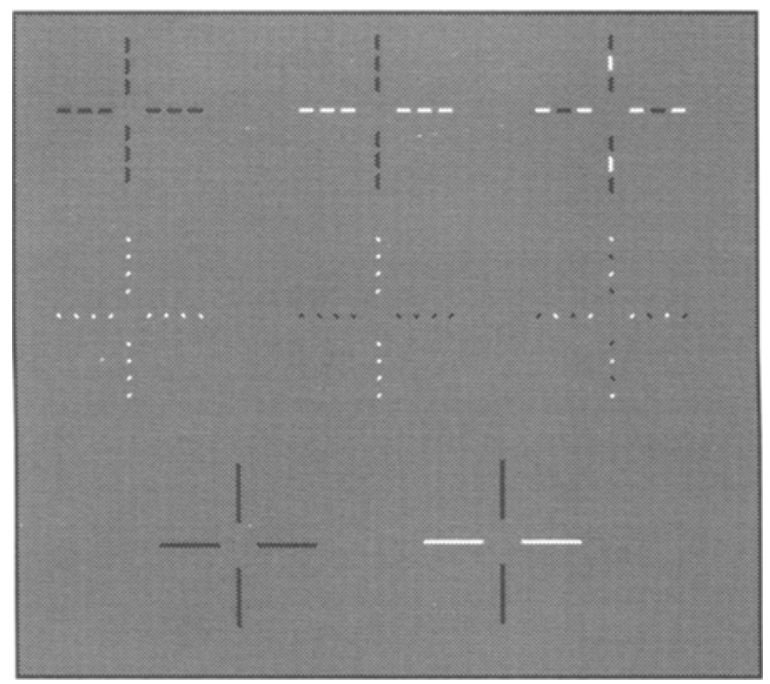

Figure 1. (A) Different versions of the Ehrenstein illusion. A bright diamond, square, or circle is perceived in the center of the configurations despite the absence of a difference in luminance between the apparent figure and the background. (B) Ehrenstein configurations made of continuous lines, dashed lines, or dotted lines were presented in our experiments. The contrast polarity and the spacing of the individual figure elements were varied to obtain contrast illusions of varying subjective magnitude. Generally, configurations with continuous lines yielded the strongest, and configurations with largely spaced dots yielded the weakest, subjective magnitudes.

collinear dashes, which seem to be integrated better than collinear dots. The combinations between inducer type (continuous, dashes, and dots), spacing, and polarity used in the Ehrenstein configuration led to frequencies of perception ranging from $0 \%$ to $100 \%$.

In the following experiments, we used some of the illusory figures from that study and investigated the effects on three different psychophysical indicators: subjective magnitude (rating), response time, and frequency of perception or discriminability of the brightness percept. Subjective magnitude was determined in a first experiment by a cross-modality matching procedure like the one used by Dresp, Lorenceau, and Bonnet (1990) in a study of the perception of apparent brightness in the Kanizsa square. In a second experiment, we measured the time necessary to perceive the presence or the absence of an apparent surface in these same illusory figures, replicating the procedure used by Dresp et al. (1996). We found that subjective magnitude, response time, and frequency of perception provide complementary data reflecting different aspects of information processing involved in the perception of brightness illusions.

\section{EXPERIMENT 1}

The first experiment assessed the subjective magnitude of the brightness illusion perceived in the different Ehrenstein figures presented here. These findings will be used for comparison with the data from a second experiment in which response times were recorded in a discrimination task.

\section{Method}

Subjects. Seven observers participated in the experiment, ranging in age from 20 to 40 years. Some of them were familiar with illusory figures, others not. All subjects, however, were naive to the purpose of the study. Two of them can be regarded as psychophysically trained. All observers had normal or corrected-to-normal vision.

\section{Stimuli}

The stimuli used were Ehrenstein figures with either four inducing lines or four inducers made of either aligned dashes or aligned dots (see Figure 1B for an illustration). The polarity of contrast of the inducing elements could be uniform or alternating. In the case of uniform polarity, the inducers were either all black or all white. In the case of alternating polarity, the two vertical inducing lines were of the same polarity, and the horizontal lines were of the other polarity. In the configurations consisting of aligned dashes or dots, contrast polarity of the individual inducing elements was varied either between or within inducing lines.

The luminance of the gray background upon which the stimuli were presented was $12 \mathrm{~cd} / \mathrm{m}^{2}$. The luminance of the white inducing elements was about $22 \mathrm{~cd} / \mathrm{m}^{2}$ and that of the black inducers about $2 \mathrm{~cd} / \mathrm{m}^{2}$. With these values, the stimulus contrast in the black version of a configuration with continuous lines was physically equal to the stimulus contrast of the white version. In figures with white inducers, the illusory region appeared darker than, and in stimuli with black inducers it appeared lighter than, the background.

In the figures with continuous inducing lines, the size of the gap separating the lines was varied. Gap sizes were about $6,12,18,24$, and $30 \mathrm{arcmin}$. The width (about 2 arcmin) and the length of the lines (about $1^{\circ}$ ) were constant. In the figures made of aligned dashes and dots, the gap in the center of the configurations was held constant at 6 arcmin, and the space between dashes and dots was varied instead. Interelement spaces of $9,12,15,18$, and 38 arcmin were generated. The dots had a constant diameter of $2 \mathrm{arcmin}$, and the width (also $2 \mathrm{arcmin}$ ) and the length ( $4 \mathrm{arcmin}$ ) of the dashes in the other figures were also held constant. Altogether, 65 different stimulus configurations with varying subjective magnitude were presented to the 7 observers. In earlier experiments (Dresp et al., 1996), such figures were used to study illusory brightness perception as a function of local variations in stimulus geometry, with an emphasis on assumptions concerning the underlying feature integration 
mechanisms. These figures were found to produce a variety of illusory percepts covering a wide range of different, discriminable, illusory strengths (see Dresp et al., 1996).

Procedure. The figures were generated by means of a VGATrident graphics card installed in a Hewlett Packard Vectra 486. They were presented in random order on the computer screen (Philips Monochrome, $640 * 480,60 \mathrm{~Hz}$ ), and the presentation of each figure was repeated twice within an experimental session. The observers were placed in front of the screen at a viewing distance of approximately $80 \mathrm{~cm}$ with their heads resting on a chin support. The figures appeared in the center of the screen, and the experiment was run under low photopic viewing conditions.

The observers had to determine the magnitude of the subjective difference in brightness between the center of a given configuration and the background. They were informed that it could be seen either as brighter than the background or as darker than the background according to the combination of inducer type, element spacing, and contrast polarity. A cross-modality matching task, described earlier by Stevens (1975), and applied by some of us in previous experiments on illusory brightness enhancement in Kanizsa squares (Dresp et al., 1990), was given to the subjects. They had to draw a line from a starting point on a sheet of paper; the length of the line had to reflect the strength of the brightness difference perceived in a given figure. The line could be drawn to the left of the starting point for "negative" illusions (darker central surface) and to the right for "positive" illusions (brighter central surface). Observers could look at each figure for as long as they wanted, and had to press the "enter" key to initiate the presentation of the next figure on the screen. For each figure, a separate response sheet had to be used.

\section{Results and Discussion}

The subjective magnitude reported on the response sheets was analyzed, and the length of a line drawn for a given figure was taken as a measure of the strength of the brightness illusion in this experiment. The figures made of dashes or dots the contrast polarity of which alternated within a given inducing line engendered little or no perception of brightness differences. In all the cases, they yielded rating lines shorter than $6.5 \mathrm{~mm}$.

The following analyses are concerned with the global effects of contrast polarity and inducer type. We compared the results of the 7 observers in the two polarity conditions ("same" vs. "varying" between inducers) and the three figure conditions ("continuous lines" vs. "dashes" vs. "dots") while averaging over the spacing factor. Generally, figures with inducing elements of the same contrast polarity were found to yield a stronger illusion, reflected through a stronger subjective magnitude, than figures with inducing elements of opposite contrast polarity $(32 \mathrm{~mm}>17 \mathrm{~mm})$. This effect was statistically significant $[t(14)=5.59, p<.001]$. Figures with continuous inducing lines produced significantly stronger illusions than figures with dashes, and figures with dashes did better than figures with dots $[47 \mathrm{~mm}>17 \mathrm{~mm}>10 \mathrm{~mm} ; F(2,12)=$
$23.83, p<.001]$. The global interaction between contrast polarity and figure type was not significant.

The effect of interelement spacing was assessed in separate analyses for the three types of figures (figures with continuous lines, figures with dashes, and figures with dots). For each figure type, we compared the results of the 7 observers in the two polarity conditions ("same" vs. "varying between inducers") and the five spacing conditions.

The results for figures with continuous inducing lines show that with increasing size of the gap in the center of the figures, the strength of the illusion does not change significantly. With continuous inducing lines, the illusion is strong enough to appear whatever the gap size. As expected from the global comparisons, figures with lines of the same contrast polarity produced significantly stronger illusions $[t(4)=3.51, p<.03]$. The interaction between polarity and spacing was not significant.

The results for figures with dashes show that with increasing interelement spacing, the strength of the illusion decreased significantly $[F 4,24)=7.83, p<.001]$. As expected from the global comparisons, figures with dashes of the same contrast polarity produced significantly stronger illusions $[t(4)=3.94, p<.02]$ than those with dashes the polarity of which varied from one inducing line to the other. The interaction between polarity and spacing was not significant.

The configurations made of dots generally produced weak illusions, with low subjective magnitude falling to zero when interelement spacing increased beyond a certain limit. The effect of interelement spacing on subjective magnitude was significant $[F(4,24)=9.67, p<.001]$. Stimuli with homogeneous contrast polarity did not produce a stronger illusion than those with dots the polarity of which varied from one inducing line to the other. With figures made of dots, the illusion was weak whatever the contrast polarity. As in the other figure conditions, the interaction between polarity and spacing was not significant.

The subjective magnitude varied between 0 and $76 \mathrm{~mm}$ for the length of the rating lines in the different stimuli. Table 1 shows the mean results obtained with the 65 figures. We grouped the different length values into 10 response classes, as shown in the table. For each response class, the number of corresponding stimulus figures is shown. Thirty-four out of a total of 65 figures corresponded to only one response class, namely the one with rating length values from 0 to $8 \mathrm{~mm}$. This result could indicate two things: Either almost half of the figures used here elicited rather weak or insignificant illusory percepts, or the estimation procedure induced some kind of

Table 1

Results of Experiment 1: Number of Figures Corresponding to Different Classes of Line Length Values Ranging From 0 to 80 Millimeters $(N=65)$

Classes of Responses

$\begin{array}{llllllllll}0-8 \mathrm{~mm} & 8-16 \mathrm{~mm} & 16-24 \mathrm{~mm} & 24-32 \mathrm{~mm} & 32-40 \mathrm{~mm} & 40-48 \mathrm{~mm} & 48-56 \mathrm{~mm} & 56-64 \mathrm{~mm} & 64-72 \mathrm{~mm} & 72-80 \mathrm{~mm}\end{array}$

$\begin{array}{llllllllll}34 & 8 & 3 & 8 & 3 & 3 & 1 & 1 & 3 & 1\end{array}$


criterion bias. We think that the first possibility is rather unlikely, given that the same stimuli had previously elicited a much larger variety of responses in a discrimination task (Dresp et al., 1996).

However, the relative rating length values indicate that the observations are globally consistent with those from the earlier discrimination experiment (yes/no procedure), in which the role of spacing, as well as contrast polarity of the different inducing elements on the formation of illusory surfaces, was investigated. With increasing spacing of the individual stimulus features (dashes or dots) constituting an inducing line here, the strength of illusory surface enhancement decreases. The strongest effects are perceived in figures with continuous inducing lines.

\section{EXPERIMENT 2}

The second experiment was concerned with measures of the discriminability of the brightness illusion in the same stimulus figures with an additional measure of the time necessary to decide on the presence or the absence of an illusion in a given figure. A yes/no procedure was used, and the observations were then compared with those made in the first experiment.

\section{Method \\ Subjects. The same 7 observers as in Experiment 1 took part. Three of them were run in this second experiment before doing the magnitude estimation task; the others did magnitude estimation first. \\ Stimuli. The stimuli, apparatus, and technical conditions of stimulus presentation were basically the same as in Experiment 1 \\ Procedure. A yes/no procedure was used in which the observers had to judge as quickly as possible whether or not the center of a given configuration appeared to differ in brightness relative to the background, knowing that this difference could appear as either positive or negative. Each stimulus figure was presented four times, in random order, per session and per observer. The responses were encoded by means of a response key set connected to the computer Response times were also computed. As soon as one of the two keys was struck, the figure disappeared from the screen and the next trial was initiated. The time interval between stimulus offset and the pre- sentation of the next figure was about $800 \mathrm{msec}$. Two experimental sessions were run for each subject.}

\section{Results and Discussion}

The percentage of "yes" responses and response times were taken as a measure of the relative strength of the brightness illusion in this experiment. As in the first experiment, on subjective magnitude, the figures made of dashes or dots the contrast polarity of which was alternating within a given inducing line engendered the perception of very weak brightness differences, or none at all. Except for the smallest interelement spacing, the percentage of "yes" responses was low, and the response times show little or no variation.

In the study of the global effects of contrast polarity and inducer type, we compared the results of the 7 observers in the two polarity conditions and the three fig- ure conditions while averaging over the spacing factor. As in Experiment 1, figures with inducing elements of the same contrast polarity were found to yield stronger illusions, reflected through a higher percentage of "yes" responses, than figures with inducing elements of opposite contrast polarity ( $76 \%$ "yes" $>68 \%$ "yes"). This effect was statistically significant $[t(14)=3.64, p<.003]$. Furthermore, response times were shorter for figures with elements of the same contrast polarity $(774 \mathrm{msec}<$ $817 \mathrm{msec})$. This effect was statistically significant $[t(14)=$ $2.11, p<.05]$. As in Experiment 1, figures with continuous inducing lines produced significantly stronger illusions than figures with dashes, and figures with dashes did better than figures with dots [97\% "yes" $>77 \%$ "yes" $>45 \%$ "yes"; $F(2,12)=24.40, p<.001]$. Furthermore, response times were shorter for figures with lines than for figures with dashes, and for figures with dashes than for figures with dots $[586 \mathrm{msec}<837 \mathrm{msec}<$ $944 \mathrm{msec} ; F(2,12)=15.55, p<.001]$. Global interactions between contrast polarity and figure type were not significant for either of the two dependent variables.

As in Experiment 1, the effect of interelement spacing was assessed in separate analyses for the three types of figures. For each figure type, we compared the results of the 7 observers in the two polarity conditions and the five spacing conditions.

The data for figures with continuous inducing lines show that with increasing size of the gap in the center of the configurations, the strength of the illusion did not change significantly. The same result was found in Experiment 1 with subjective magnitude. The illusion was strong whatever the gap size. However, response times increased significantly with increasing gap size $[F(4,24)=$ $4.26, p<.01]$, showing that stimulus uncertainty increases despite the apparently leveled strength of the illusory percept. Figures with lines of the same contrast polarity did not produce a significantly higher percentage of "yes" responses or shorter response times, whereas in Experiment 1 subjective magnitude was found to be significantly higher for figures containing only one contrast polarity. The interactions between polarity and spacing were not significant for either the percentage of "yes" responses or for response times.

The results for figures with dashes show that with increasing interelement spacing, the strength of the illusion decreased significantly $[F(4,24)=6.67, p<.002]$, while response times did not change significantly. Moreover, figures with dashes of the same contrast polarity produced significantly stronger illusions $[t(4)=4.08, p<.02]$ and shorter response times $[t(4)=2.86, p<.05]$ than those with dashes the polarity of which varied from one inducing line to the other. The interactions between polarity and spacing were not significant for either of the two dependent variables.

The configurations made of dots generally produced weak illusions, with low percentages of "yes" responses, falling to $10 \%$ when interelement spacing increased beyond a certain limit. The effect of interelement spacing on 
the percentage of "yes" was significant $[F(4,24)=23.49$, $p<.001]$, but the effect on response times was not. Figures with dots of the same contrast polarity did not produce significantly stronger illusions or shorter response times than figures with both polarities. The same result was observed in Experiment 1 with subjective magnitude. The interactions between polarity and spacing were not significant for either of the two dependent variables.

Thirty-one of the figures elicited illusory percepts, reflected by discrimination frequencies ranging from $0 \%$ to $50 \%$, and 34 of the figures produced illusions, reflected by discrimination frequencies ranging from $50 \%$ to $100 \%$. Table 2 shows the number of figures corresponding to the different response classes, ranging from $0 \%$ to $100 \%$ "yes" responses. The number of stimulus figures in the different response categories was more evenly spread than in the subjective magnitude experiment. Thus, the discrimination task, or frequency of perception measure, appears to have provided a more balanced, or generally finer, estimate of the relative strength of the brightness illusion in the different configurations.

Nevertheless, 22 of the figures elicited illusory percepts, reflected by discrimination frequencies ranging from $90 \%$ to $100 \%$. Data from Tables 1 and 2 are combined in Figure 2A. Each type of behavioral response (subjective magnitude, percentage of "yes" responses, and response times) appears for the 65 figures. When the illusory percept is relatively weak, subjective magnitude does not provide discriminative information about the different levels of sensation, while the percentage of "yes" responses neatly increases, and response times decrease. Nevertheless, while response times and subjective magnitude seem to reveal the differences in strength of the stronger illusions, the percentage of "yes" responses shows a ceiling effect for these stronger illusions. This suggests that magnitude information is the better indicator when the illusory strength has reached a critical level.

Figures $2 \mathrm{~B}$ and 3 show the mean results of the 7 observers. When the subjective magnitude of the different illusory percepts was plotted as a function of the percentage of "yes" responses from the discrimination procedure, the curves showed that subjective magnitude only began to increase when a rate of $50 \%$ of "yes" responses was reached (Figure 2B). This result suggests that subjective magnitude measures provide reliable estimates of illusory percepts beyond that limit only, whereas the weaker percepts appear to be better discriminated by the frequency-of-perception measure.

The response time data from Experiment 2 show that weak illusions (discrimination $<50 \%$ ) elicited gradually increasing response times when discriminability increased between $0 \%$ and $50 \%$ of "yes" responses. Furthermore, response times began to decrease systematically beyond the rate of 50\% "yes" (see again Figure 2B). Therefore, response times decrease when the brightness illusion has reached a critical level of strength, which here appears to be defined by the point where the illusory percept is discriminated in $50 \%$ of the trials. It is understood that obvious anticipatory responses (response time $<100 \mathrm{msec}$ ) were not taken into account for data analysis (this corresponds to never more than four responses per observer in total).

A separate analysis of response times for "yes" responses and response times for "no" responses reveals the intricacies of the perceptual processes under investigation even better. When response times are analyzed as a function of the percentage of "yes" responses (Figure 3), we see that response times for "no" are shorter than response times for "yes," with a little increase for both response types when the strength of the illusory percept has not yet reached the $50 \%$ discrimination limit. Beyond that limit, response times for "no" responses were found to increase further, whereas response times for "yes" responses immediately began to decrease. This finding indicates that discriminability and processing time interact in the decision process underlying illusory percepts.

\section{GENERAL DISCUSSION}

The results from our experiments show that in brightness stimuli with a wide range of discriminable phenomenal strengths, the joint measure of subjective magnitude, frequency of perception, and processing time may help to tackle the different underlying perceptual processes. In particular, it appears that response times and the frequency of perceiving a brightness illusion as a function of the configurational context cannot replace the classic magnitude estimation approach, and vice versa. The three measures taken together reveal complementary aspects of information processing and lead to a satisfactory and coherent body of data in the assessment of the relative discriminability of illusory and similar subjective phenomena. The complementary nature of the different behavioral observations can be summarized as follows.

In configurations where the brightness illusion is weak, the discrimination task appears to provide finer estimates of the relative strength of the phenomenon than does the scaling task. When the uncertainty about the presence of an illusion is high, subjective magnitude is leveled,

Table 2

Results of Experiment 2: Number of Figures Corresponding to the Different Response Classes Ranging From 0\% to $100 \%$ "yes" Responses $(N=65)$

\begin{tabular}{cccccccccccc}
\hline \multicolumn{10}{c}{ Classes of Responses } \\
\hline $0 \%-10 \%$ & $10 \%-20 \%$ & $20 \%-30 \%$ & $30 \%-40 \%$ & $40 \%-50 \%$ & $50 \%-60 \%$ & $60 \%-70 \%$ & $70 \%-80 \%$ & $80 \%-90 \%$ & $90 \%-100 \%$ \\
\hline 4 & 8 & 9 & 6 & 4 & 3 & 4 & 2 & 3 & 22 \\
\hline
\end{tabular}


A

- Percentage "yes" - Response times (ms)

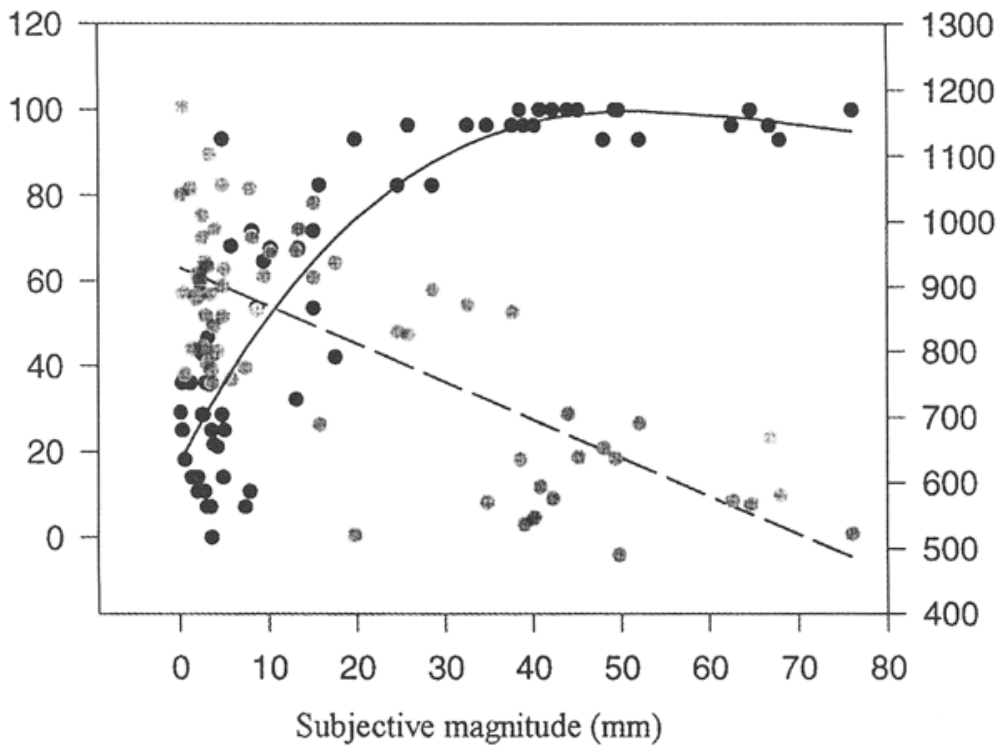

B

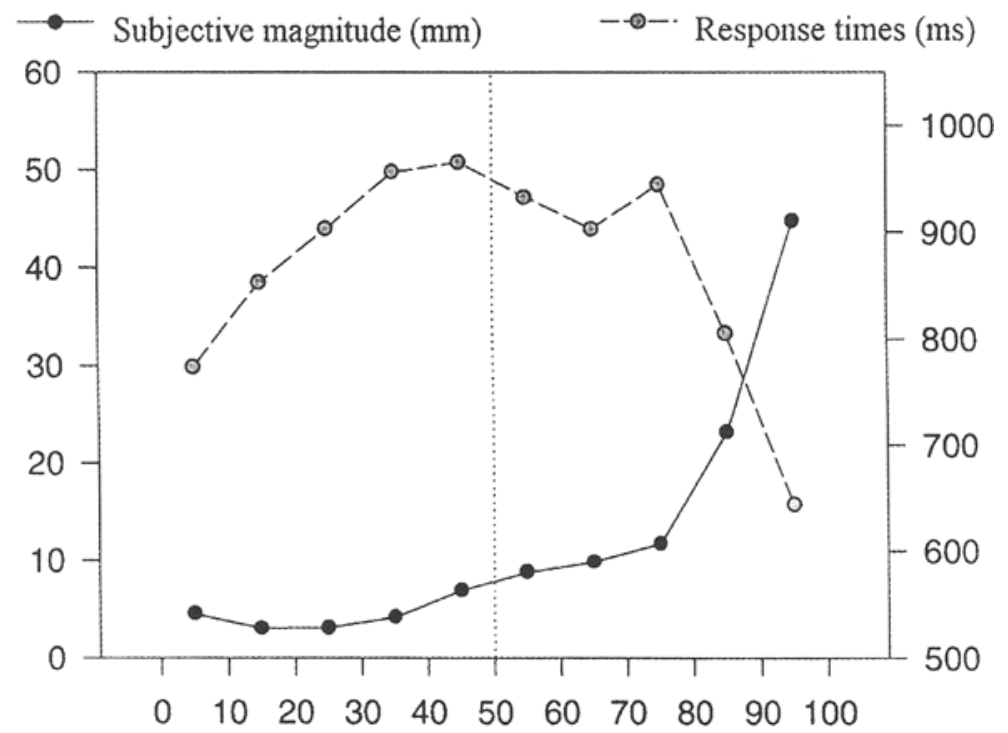

Percentage "yes"

Figure 2. (A) Response times and frequency of the perception of brightness difference as a function of the subjective magnitude for each of the 65 figures. (B) Subjective magnitude (data from Experiment 1) and response times (data from Experiment 2) as a function of the discriminability of the contrast illusion as reflected through the percentage of "yes" responses in a yes/no task (data from Experiment 2). The graphs show mean data of 7 naive observers.

whereas the percentage of "yes" increases and response times decrease. On the other hand, whereas response times and subjective magnitude distinctively reveal the increasing strength of the illusion at higher certainty levels, the percentage of "yes" responses quickly reaches a ceiling effect there. These observations lead to the con- clusion that the frequency-of-perception measure is more reliable for stimuli about which the uncertainty about the presence or the absence of an illusion is high.

Complementarily, the data show that subjective magnitude begins to increase only when a rate of $50 \%$ of the responses measured in the yes/no task is reached. This 


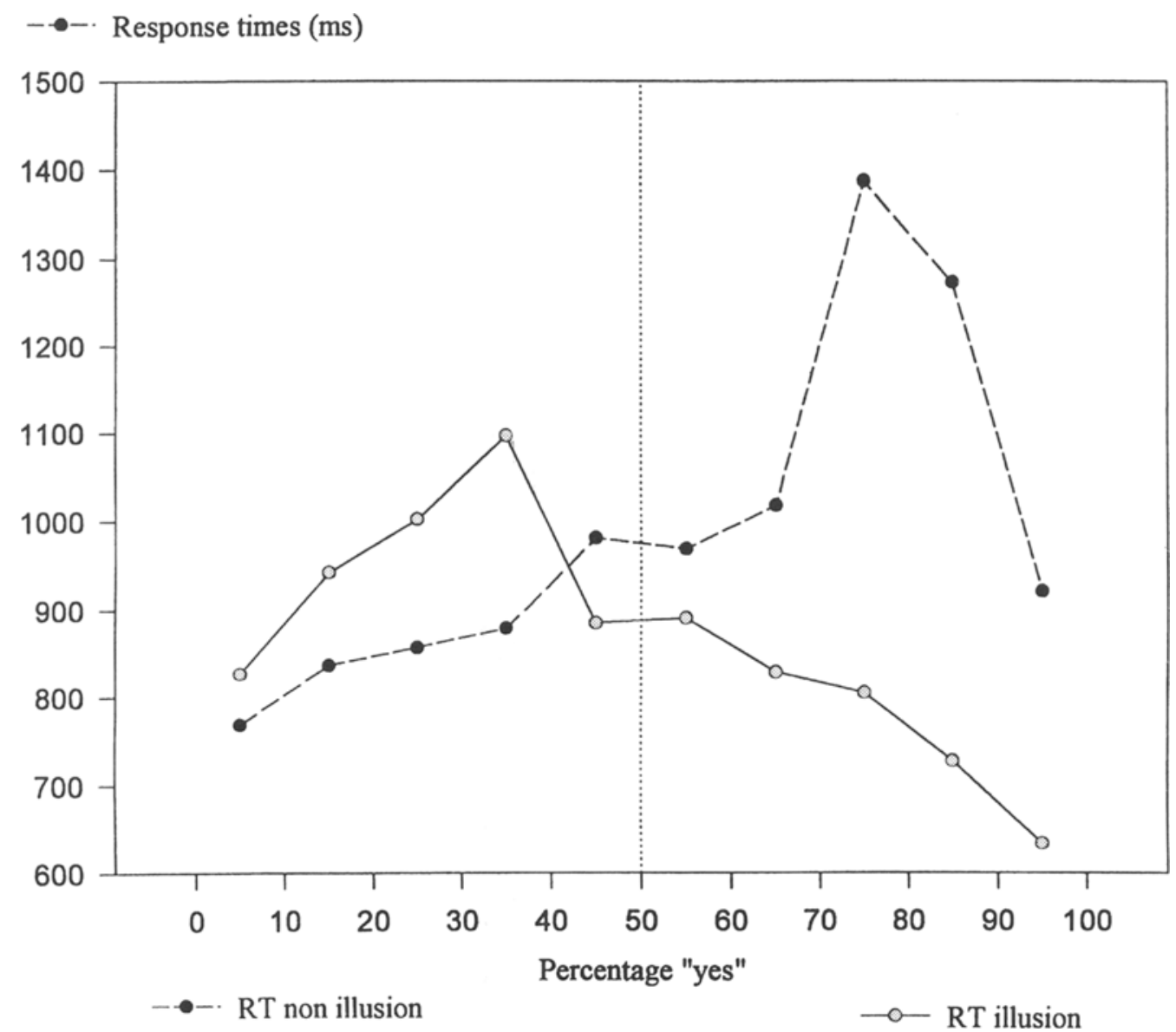

Figure 3. Response times for "yes" responses and response times for "no" responses in the discrimination task (Experiment 2 ) as a function of the discriminability of the contrast illusion.

result suggests that the subjective magnitude measure provides more reliable estimates of relatively strong sensations - that is, when uncertainty about the presence or the absence of an illusion is low.

Weak illusions (discrimination $<50 \%$ ) elicit gradually increasing response times when discriminability increases, while response times begin to decrease systematically when $50 \%$ of the responses from the discrimination task are positive-that is, when the brightness sensation has reached some kind of critical level of intensity, apparently defined by the point where the illusion is discriminable in $50 \%$ of the trials and also where subjective magnitude begins to increase. We speculate that this level of sensation reflects a state of observation at which the uncertainty about the stimulus, or about the presence of a brightness illusion, is the highest. This, in turn, would also explain why response times are the longest at precisely this level. The variations in response times observed in this experiment would then reflect (1) an increase in the uncertainty about the absence of an illusion for discrimination levels below $50 \%$ and (2) an increase in the certainty about the presence of an illusion for discrimination levels above $50 \%$. This is reflected by the observation that the discriminability of the illusion and processing time appear to be related by an inverted U-shape function.

The decrease in response times when the subjective magnitude increases and the frequency of perception is more than $50 \%$ lead to a comparison of the phenomena observed with brightness illusions with those obtained with real brightness sensations. Indeed, discrimination experiments with luminous forms have shown that response times decrease when the physical luminance of the stimulus increases up to a certain level, beyond which a ceiling effect occurs (see, e.g., Pins \& Bonnet, 1996, 1997). Therefore, it may be that "illusory" brightness beyond the $50 \%$ discrimination limit produces effects on processing time similar to those produced by "real" brightness.

Finally, the analyses of response times for "no" and for "yes" suggest that the frequency measure and processing time are related parameters. Their joint analysis reveals the decision process involved in the perception of illusions with largely varying phenomenal strength. If we assume that relatively longer response times reflect relatively higher stimulus uncertainty, which is a conse- 
quence of the relatively weaker brightness sensations, then the increase in response times for "no" responses when subjective magnitude increases and, consequently, the decrease in response times for "yes" responses may, as suggested above, be interpreted in terms of an increase in the subject's certainty that there is an illusion. When a sufficiently high degree of stimulus certainty is reached, response times for both "yes" and "no" responses become similar, and very short.

The three perceptual measures reported in this paper are commonly used separately to study brightness illusions like the one that occurs in the Ehrenstein configuration. Comparisons between these different measures show that the use of only one of them cannot replace the others because the three measures are not equally informative with regard to the different levels of information processing that are involved. It seems important to take advantage of the complementarity of these measures to study the perceptual genesis of illusory phenomena and their dynamic properties. Some computational models of perceptual organization make predictions regarding the spatial and temporal dynamics of preattentive and attentive processes underlying visual illusions (e.g., Elder \& Zucker, 1998; Grossberg \& Mingolla, 1985). Some of these dynamics can be tested in a reaction time study, especially aspects of temporal processing related to visual attentional mechanisms. The organization of boundary (contour signals) and surface (brightness signals) representations within Grossberg's (e.g., 1997) form vision model, for example, suggests working hypotheses that clarify how preattentive visual spatial mechanisms may interact with attentive processes in the emergence of 3-D percepts from 2-D pictures. The perception of a 3-D surface is an important aspect of the Ehrenstein illusion, and its genesis most likely involves attentional processes. Strong illusory brightness phenomenally produces instant pop-out of the 3-D percept, whereas weaker brightness illusions are much less striking. In visual search tasks, surfaces that pop out have been found to yield shorter response times than less striking ones (e.g., Treisman \& Gor- mican, 1988). The involvement of attentional factors in the perception of illusory stimuli can therefore be brought to the fore by reaction time measures; it cannot be investigated on the sole basis of magnitude estimates.

\section{REFERENCES}

Dresp, B., LorenceaU, J., \& Bonnet, C. (1990). Apparent brightness enhancement in the Kanizsa square with and without illusory contour formation. Perception, 19, 483-489.

Dresp, B., Salvano-Pardieu, V., \& Bonnet, C. (1996). Illusory form with inducers of opposite contrast polarity: Evidence for multistage integration. Perception \& Psychophysics, 58, 111-124.

EldER, J. H., \& ZUCKER, S. W. (1998). Evidence for boundary specific grouping. Vision Research, 328, 143-152.

GrossberG, S. (1997). Cortical dynamics of 3-D figure-ground perception of 2-D pictures. Psychological Review, 104, 618-658.

GrossberG, S., \& Mingolla, E. (1985). Neural dynamics of form perception: Boundary completion, illusory figures, and neon color spreading. Psychological Review, 92, 173-211.

Lesher, G. W., \& Mingolla, E. (1993). The role of edges and lineends in illusory contour formation. Vision Research, 33, 2253-2270.

PiNs, D., \& BonNET, C. (1996). On the relation between stimulus intensity and processing time: Piéron's law and choice reaction time. Perception \& Psychophysics, 58, 390-400.

PINS, D., \& BonNET, C. (1997). Reaction times reveal the contribution of the different receptor components in luminance perception. $P s y$ chonomic Bulletin \& Review, 4, 359-366.

Shipley, T. F., \& Kellman, P. J. (1992). Strength of visual interpolation depends on the ratio of physically specified to total edge length. Perception \& Psychophysics, 52, 97-106.

SPILlmaNN, L., \& DresP, B. (1995). Phenomena of illusory form: Can we bridge the gap between levels of explanation? Perception, 24, 1333-1364.

Spillmann, L., Fuld, K., \& Neumeyer, C. (1984). Brightness matching, brightness cancellation, and increment threshold in the Ehrenstein illusion. Perception, 13, 513-520.

STEVENS, S. S. (1975). Psychophysics: Introduction to its perceptual, neural, and social prospects. New York: Wiley.

Treisman, A. M., \& GormiCaN, S. (1988). Feature analysis in early vision: Evidence from search asymmetries. Psychological Review, 95, $15-48$.

ZuCKer, S. W., \& Davis, S. (1988). Points and end-points: A size/spacing constraint for dot grouping. Perception, 17, 229-247.

(Manuscript received January 20, 1998; revision accepted for publication August 31, 1998.) 